PROCEEDINGS OF THE

AMERICAN MATHEMATICAL SOCIETY

Volume 140, Number 4, April 2012, Pages 1125-1132

S 0002-9939(2011)10894-3

Article electronically published on November 16, 2011

\title{
ON THE CANONICAL DECOMPOSITION OF GENERALIZED MODULAR FUNCTIONS
}

\author{
WINFRIED KOHNEN AND GEOFFREY MASON
}

(Communicated by Kathrin Bringmann)

\begin{abstract}
The authors have conjectured that if a normalized generalized modular function (GMF) $f$, defined on a congruence subgroup $\Gamma$, has integral Fourier coefficients, then $f$ is classical in the sense that some power $f^{m}$ is a modular function on $\Gamma$. A strengthened form of this conjecture was proved in case the divisor of $f$ is empty. In the present paper we study the canonical decomposition of a normalized parabolic GMF $f=f_{1} f_{0}$ into a product of normalized parabolic GMFs $f_{1}, f_{0}$ such that $f_{1}$ has unitary character and $f_{0}$ has empty divisor. We show that the strengthened form of the conjecture holds if the first "few" Fourier coefficients of $f_{1}$ are algebraic. We deduce proofs of several new cases of the conjecture, in particular if either $f_{0}=1$ or the divisor of $f$ is concentrated at the cusps of $\Gamma$.
\end{abstract}

\section{INTRODUCTION}

Let $\Gamma \subset \Gamma_{1}:=\mathrm{SL}_{2}(\mathbb{Z})$ be a congruence subgroup and let $\mathcal{H}$ be the complex upper half-plane. We consider generalized modular functions of weight zero (GMFs) on $\Gamma$. These are holomorphic functions $f: \mathcal{H} \rightarrow \mathbb{C}$ which satisfy

$$
f(\gamma \circ z)=\chi(\gamma) f(z) \quad(\forall \gamma \in \Gamma)
$$

where $\chi: \Gamma \rightarrow \mathbb{C}^{*}$ is a (not necessarily unitary) character, and which are meromorphic at the cusps. We call $f$ a parabolic generalized modular form (PGMF) if it also satisfies $\chi(\gamma)=1$ for all parabolic elements $\gamma \in \Gamma$ of trace 2. If $f$ is a GMF, then some power $f^{m}$ of $f$ is a PGMF. For further details we refer to [KM1]. In this paper we deal mainly with PGMFs.

At the cusp at infinity, a PGMF $f$ has a Fourier expansion

$$
f(z)=\sum_{n \geq h} a(n) q_{N}^{n} \quad\left(0<\left|q_{N}\right|<\epsilon\right)
$$

for appropriate $h \in \mathbb{Z}, N \in \mathbb{N}$ and where $q_{N}:=e^{2 \pi i z / N} \quad(z \in \mathcal{H})$.

We shall call $f$ normalized if $a(h)=1$.

According to [KM2], each normalized PGMF $f$ on $\Gamma$ has a canonical decomposition

$$
f=f_{1} f_{0},
$$

Received by the editors August 12, 2010 and, in revised form, November 9, 2010. 2000 Mathematics Subject Classification. Primary 11F03, 11F99, 17B69.

Key words and phrases. Canonical decomposition, generalized modular function.

The second author was supported in part by the NSF and NSA.

(C)2011 American Mathematical Society 
where $f_{1}$ and $f_{0}$ are normalized PGMFs on $\Gamma, f_{1}$ has unitary character, and the divisor of $f_{0}$ is empty. The canonical decomposition is indeed unique, which amounts to the assertion [KM2] that a PGMF with empty divisor and unitary character is constant. Note that it follows from these conditions and our assumptions that

$$
f_{1}=q_{N}^{h}+\ldots, \quad f_{0}=1+\ldots .
$$

It was conjectured in $\mathrm{KoM}$, Section 1 , that if a normalized PGMF $f$ on the Hecke congruence subgroup $\Gamma_{0}(N)$ of level $N$ has integral Fourier coefficients, then it must be classical; i.e. the character $\chi$ is of finite order. A proof of this conjecture would have some very important consequences in rational conformal field theory, as explained in $\underline{\mathrm{KoM}}$.

In the present paper we shall show that the conjecture is equivalent to requiring that the first "few" Fourier coefficients of the function $f_{1}$ in the decomposition (1) are algebraic numbers. We will actually prove slightly stronger statements regarding the hypothesis on the Fourier coefficients of $f$, requiring only that they are rational and $p$-integral for almost all primes $p$. This result implies several new cases of the conjecture: if $\chi$ is unitary (i.e. $f_{0}=1$ ), or if the $\operatorname{divisor} \operatorname{div}(f)$ of $f$ is supported at the cusps of $\Gamma$. Some special cases of the second assertion, including the case when $\operatorname{div}(f)$ is empty (i.e. $f_{1}=1$ ), were established in $[\mathrm{KoM}$. Our present results are valid for an arbitrary congruence subgroup $\Gamma$.

Apart from the decomposition (1), there are two main ingredients to the proof of our results: the first is a theorem of Scholl-Waldschmidt ([Sc, $\mathrm{W}$ ) on the transcendence of canonical differentials of the third kind on modular curves; the second one is the result on PGMFs with empty divisors $\mathrm{KoM}$ already mentioned above, whose proof largely depends on the analytic theory of Dirichlet series.

The paper is organized as follows. We give the proof of the main results, Theorem 2.1 and Corollary 2.2. in Section 3. In Section 4 we consider the action of complex conjugation on PGMFs and their characters. For example, we show that if the Fourier coefficients of a PGMF $f$ are real, then so are those of $f_{0}$ and $f_{1}$.

\section{Statement of RESUlts}

We define

$$
\kappa_{\Gamma}:=\left[\frac{1}{6}\left[P \Gamma_{1}: P \Gamma\right]\right]+1-c_{\Gamma} .
$$

Here $P \Gamma_{1}:=\mathrm{PSL}_{2}(\mathbb{Z})$ and $P \Gamma$ denotes the image of $\Gamma$ under the natural projection $\Gamma_{1} \rightarrow P \Gamma_{1}$. Furthermore $[x](x \in \mathbb{R})$ denotes the greatest integer function and $c_{\Gamma}$ is the number of cusps of $\Gamma$.

The main result of the paper is the following:

Theorem 2.1. Let $f$ be a normalized PGMF on $\Gamma$ whose Fourier coefficients a $(n)$ are rational for all $n$ and are $p$-integral for all but a finite number of primes $p$. Let (1) be the canonical decomposition of $f$. Then the character $\chi$ of $f$ is of finite order if, and only if, the Fourier coefficients $a_{1}(n)$ of $f_{1}$ are algebraic for $h \leq n \leq \kappa_{\Gamma}+h$.

Theorem 2.1 has the following consequence.

Corollary 2.2. Let $f$ be a PGMF on $\Gamma$ and let $\chi$ be the character of $f$. Assume that the Fourier coefficients $a(n)$ are rational for all $n$ and are $p$-integral for all 
but a finite number of primes $p$. Then $\chi$ has finite order if either of the following conditions holds:

(a) $\chi$ is unitary.

(b) The divisor of $f$ is concentrated at the cusps of $\Gamma$.

We point out ([KM1]) that the condition on the divisor in part (b) is equivalent to the assumption that the logarithmic derivative $f^{\prime} / f$, which is generally a meromorphic modular form of weight 2 on $\Gamma$, is in fact holomorphic.

\section{Proof of Theorem 2.1}

In one direction the conclusion is easy. Indeed, assume that $\chi$ has finite order $m$. Then $f^{m}$ has trivial character, so that

$$
f^{m}=f_{1}^{m} \cdot f_{0}^{m}=f_{1}^{m} \cdot 1
$$

must be the canonical decomposition of $f^{m}$. Therefore $f$ and $f_{1}$ differ by an $m$ th root of unity, and since they are both normalized, then they are equal and all Fourier coefficients of $f_{1}$ are algebraic, indeed rational.

In the other direction we may, and from now on we shall, assume without loss of generality that $\Gamma=\Gamma(N)$ is the principle congruence subgroup of $\Gamma_{1}$ of level $N$. Indeed, if $\Gamma$ contains the normal subgroup $\Gamma(N)$, and if $\chi^{r}=1$ on $\Gamma(N)$, then $\chi^{r e}=1$ on $\Gamma$, where $e=\left|\Gamma_{1}: \Gamma(N)\right|$ is the index.

Now suppose that $a_{1}(n)$ is algebraic for $h \leq n \leq \kappa+h$, where we have abbreviated $\kappa:=\kappa_{\Gamma}$. Solving recursively in (1) for the Fourier coefficients $a_{0}(n)$ of $f_{0}$, we see that our assumption implies that each $a_{0}(n)(0 \leq n \leq \kappa)$ is also algebraic.

Let

$$
\frac{2 \pi i}{N} g_{0}:=\frac{f_{0}^{\prime}}{f_{0}}
$$

be the logarithmic derivative of $f_{0}$ and write

$$
g_{0}=\sum_{n \geq 1} b_{0}(n) q_{N}^{n} .
$$

Then we see that each $b_{0}(n)(1 \leq n \leq \kappa)$ is algebraic. Note that $g_{0}$ is a cusp form of weight 2 on $\Gamma$ with trivial character, $\operatorname{since} \operatorname{div}\left(f_{0}\right)=\emptyset$ [KM1].

We now assert

Lemma 3.1. All Fourier coefficients $b_{0}(n)(n \geq 1)$ are contained in a finite exten$\operatorname{sion} K / \mathbb{Q}$.

Proof. Our claim is essentially well known and follows from linear algebra combined with the valence formula and the fact that $\Gamma=\Gamma(N)$. However, for the reader's convenience we will give a detailed proof.

First recall that the valence formula says that the sum of the orders (measured in the appropriate local variables) of a nonzero cusp form of weight 2 on $\Gamma$ on the complete modular curve $X_{\Gamma}:=\overline{\Gamma \backslash \mathcal{H}}$ is equal to $\left[\frac{1}{6}\left[P \Gamma_{1}: P \Gamma\right]\right]$.

Since $\Gamma=\Gamma(N)$, it is well known that the space $S_{2}(\Gamma)$ of cusp forms of weight 2 for $\Gamma$ has a basis $\left\{g_{1}, \ldots, g_{d}\right\}$ of functions with rational (in fact integral) Fourier coefficients ([Sh], Thm. 3.52). Note that the valence formula implies that $d \leq \kappa$.

We write $g_{0}$ as a linear combination of the $g_{\nu}(1 \leq \nu \leq d)$. Bearing in mind that the first $\kappa$ Fourier coefficients of $g_{0}$ are algebraic, our claim will follow if we 
can show that the $\kappa \times d$ matrix $A$ whose columns consists of the first $\kappa$ Fourier coefficients of $g_{1}, \ldots, g_{d}$ has maximal rank.

To show this we argue as follows. Let $\langle$,$\rangle denote the usual inner product on$ $S_{2}(\Gamma)$. Let $P_{n}(n \geq 1)$ be the $n$-th "Poincaré series" in $S_{2}(\Gamma)$ with respect to $\langle$,$\rangle ;$ i.e., $P_{n}$ is the dual of the functional that sends a cusp form $g \in S_{2}(\Gamma)$ to its $n$-th Fourier coefficient $a_{g}(n)$. By the valence formula, $\left\{P_{1}, \ldots, P_{\kappa}\right\}$ generates $S_{2}(\Gamma)$. Hence, there exists a basis $\left\{P_{n_{1}}, \ldots, P_{n_{d}}\right\}$, where $1 \leq n_{\nu} \leq \kappa$ for all $\nu$.

On the other hand, let $\ell \in S_{2}^{*}(\Gamma)$ be any functional. Then by standard duality, there exists $L \in S_{2}(\Gamma)$ such that $\langle g, L\rangle=\ell(g)$ for all $g$. Writing $L$ in terms of the basis $\left\{P_{n_{1}}, \ldots, P_{n_{d}}\right\}$, we see that $\ell$ is a linear combination of $a\left(n_{1}\right), \ldots, a\left(n_{d}\right)$; hence the latter functionals form a basis of $S_{2}^{*}(\Gamma)$.

From the above it follows that the $\kappa \times d$ matrix $B$ whose columns are the first $\kappa$ Fourier coefficients of $P_{n_{1}}, \ldots, P_{n_{d}}$ has maximal rank (the rows with indices $n_{1}, \ldots, n_{d}$ are linearly independent). Hence the same is true for $A$, since $A$ is obtained from $B$ by multiplying with an invertible $d \times d$ matrix. This completes the proof of the lemma.

In (2) we now solve recursively for the $a_{0}(n)$. Using Lemma 3.1, we see that $a_{0}(n) \in K$ for all $n$. Therefore by (1), each $a_{1}(n)$ also lies in $K$.

We put

$$
\frac{2 \pi i}{N} g_{1}:=\frac{f_{1}^{\prime}}{f_{1}}
$$

Then $g_{1}$ also has Fourier coefficients in $K$, and $g_{1}$ is a meromorphic modular form of weight 2 on $\Gamma$ with trivial character. It has at worst simple poles in $\mathcal{H}$ with integral residues and is holomorphic at the cusps KM1.

We let

$$
D:=\operatorname{div}(f) \text {. }
$$

Then $\operatorname{deg} D=0$ [KM1, while $\operatorname{div}(f)=\operatorname{div}\left(f_{1}\right)$ by hypothesis.

The form $g_{1}$ defined by (3) gives rise to an abelian differential of the third kind,

$$
\omega_{1}:=\frac{2 \pi i}{N} g_{1} d z
$$

on $X_{\Gamma}$ with residue divisor $D$.

Lemma 3.2. The divisor $D$ is defined over a number field.

Proof. This essentially is well known: indeed, the Galois group operates on meromorphic differentials and this operation is compatible with the formation of residue divisors. However, for the convenience of the reader, we again give a detailed proof. We shall prove somewhat more, namely that each point $P$ in the support of $D$ is already fixed by $\operatorname{Gal}(\overline{\mathbb{Q}} / L)$ where $L / \mathbb{Q}$ is a finite extension.

The modular curve $X_{\Gamma}$ is defined over a number field $F$ (in fact, over $\mathbb{Q}\left(\zeta_{N}\right)$, where $\left.\zeta_{N}=e^{2 \pi i / N}\right)$. Since the cusps of $\Gamma$ are defined over a number field, we may suppose that $P$ is contained in the "open part" $Y_{\Gamma}:=X_{\Gamma} \backslash\{$ cusps $\}$ of the modular curve.

The function field $F_{X_{\Gamma}}$ of $X_{\Gamma} / F$ is a finite extension of $F(j)$, where $j$ is the classical modular invariant. By the "theorem of the primitive element" there exists a modular function $t$ for $\Gamma$ such that $F_{X_{\Gamma}}=F(j, t)$ and $t$ satisfies an algebraic equation over $F(j)$. The points of $Y_{\Gamma}$ then can be parametrized as $(j(z), t(z))$ $(z \in \Gamma \backslash \mathcal{H})$. 
Suppose that $P$ "corresponds" to $(j(z), t(z))(z \in \mathcal{H})$. It is sufficient to show that $j(z)$ is algebraic over $\mathbb{Q}$. Because $P$ is not a cusp, then $z$ is necessarily a zero of $f$, and hence a simple pole of $g_{1}$. Let

$$
G_{1}:=\frac{g_{1}^{6}}{\Delta}
$$

where $\Delta$ is the usual discriminant function of weight 12 on $\Gamma_{1}$. Then $G_{1}$ is a modular function on $\Gamma$ with a pole at $z$. Because $g_{1}$ has Fourier coefficients in $K$, the same is true of $G_{1}$. Observe that each of the translates

$$
\left(G_{1} \mid \gamma\right)(z):=G_{1}\left(\frac{a z+b}{c z+d}\right), \quad \gamma=\left(\begin{array}{cc}
a & b \\
c & d
\end{array}\right) \in \Gamma_{1},
$$

also has Fourier coefficients in a number field. Once again, this is a standard result. The proof is based on the " $q$-expansion principle" for congruence subgroups ( $[\mathrm{DR}$, VII, 4.8). (A more general proof that works for noncongruence subgroups can be found in $\mathrm{KL}$, Proposition A1.)

We may, and shall, choose $c \in \mathbb{Q}$ such that none of the modular functions $\left(G_{1} \mid \gamma\right)(z)-c$ has a zero at $z$. Now consider the "norm"

$$
\prod_{\gamma \in P \Gamma \backslash P \Gamma_{1}}\left(G_{1}-c\right) \mid \gamma \text {. }
$$

It is a modular function on $\Gamma_{1}$ with coefficients in $K$, and hence is a rational function $A(j) / B(j)$ with $A(j), B(j) \in K[j]$. By construction there is a pole at $z$, so that $B(j(z))=0$. The algebraicity of $j(z)$ follows, and the proof of the lemma is complete.

Next, observe that $\omega_{1}$ is a canonical differential on $X_{\Gamma}$; i.e.

$$
\Re\left(\int_{\sigma} \omega_{1}\right)=0
$$

for all $\sigma \in H_{1}(U ; \mathbf{Z})$, where $U:=X_{\Gamma} \backslash \operatorname{supp} D$. Indeed, this is equivalent to our assumption that $f_{1}$ has unitary character, bearing in mind the relation (3). We may now invoke an important theorem of Scholl-Waldschmidt [Sc], [W] on the transcendence of abelian differentials of the third kind on modular curves: since $g_{1}$ has Fourier coefficients in a number field, $D$ has finite order in the divisor class group. Thus there exists $m \in \mathbb{N}$ and a modular function $h_{1}$ on $\Gamma$ such that

$$
\operatorname{div}\left(\frac{f_{1}^{m}}{h_{1}}\right)=\emptyset \text {. }
$$

Therefore $f_{1}^{m}=h_{1}$, since $f_{1}$ and $h_{1}$ both have a unitary character.

Lemma 3.3. Let $X$ be a projective algebraic variety defined over a number field $F$, and let $D$ be a divisor on $X$ defined over $F$. If there is a rational function $\Psi \in \mathbb{C}(X)$ over $\mathbb{C}$ with divisor $D$, then there is a rational function $\Psi_{0} \in F(X)$ over $F$ with divisor $D$ and a constant $c \in \mathbb{C}^{*}$ such that $\Psi=c \Psi_{0}$.

Proof. We consider the linear system of $-D$ over $\mathbb{C}$, i.e. the complex vector space $L(-D)=\left\{f \in \mathbb{C}(X)^{*} \mid \operatorname{div}(f) \geq D\right\} \cup 0$. By our assumption $\Psi \in L(-D)$, and since $X$ is projective, then $L(-D)=\mathbb{C} \Psi$.

Since $D$ is defined over $F, L(-D)$ is invariant under the action of $\Gamma=\operatorname{Aut}(\mathbb{C} / F)$, the automorphism group of the field extension $\mathbb{C} / F$. We have to show that $L(-D)$ contains a $\Gamma$-invariant vector. This, however, follows from a standard descent result; 
see e.g. Lemma 16.6 in $\mathrm{Mi}$. In this lemma take $k=F, \Omega=\mathbb{C}, V=F(X)$ the field of rational functions of $X / F$, and $V(\Omega)=\mathbb{C}(X)$ the field of rational functions of $X$ over $\mathbb{C}=V \otimes_{F} \mathbb{C}, W=L(-D)$.

If we apply Lemma 3.3 in our situation (with $X=X_{\Gamma}$ ) and use the $q$-expansion principle [Mi] then we see that we can normalize $h_{1}$ to have Fourier coefficients in the compositum of $\mathbb{Q}\left(\zeta_{N}\right)$ and the field of definition of the algebraic divisor $D$ (cf. Lemma 3.2) and to have leading nonzero term equal to 1. From (1) we now obtain

$$
f^{m}=h_{1} \cdot h_{0},
$$

where

$$
h_{0}:=f_{0}^{m} .
$$

Since $\operatorname{div}\left(h_{0}\right)=\emptyset$ and $h_{1}$ has trivial character, it follows that (4) is the canonical decomposition of $f^{m}$.

Lemma 3.4. Let $h=\sum b(n) q_{N}^{n}$ be a modular function on $\Gamma=\Gamma(N)$ with Fourier coefficients in a number field, and let $\sigma \in \operatorname{Gal}(\overline{\mathbb{Q}} / \mathbb{Q})$. Then $h^{\sigma}:=\sum b(n)^{\sigma} q_{N}^{n}$ is a modular function on $\Gamma$.

Proof. This is well known. Indeed, arguing as in the proof of Lemma 3.2, we see that $j(z)$ is algebraic if $z \in \mathcal{H}$ is a pole of $h$. We therefore conclude that there exists a polynomial $P$ with algebraic coefficients and a large positive integer $M$ such that

$$
H:=\Delta^{M} P(j) h
$$

is a cusp form of weight $k \geq 2$ on $\Gamma$. The latter space has a basis of functions with rational Fourier coefficients $[\underline{S h}$, so that $H$ is a $\overline{\mathbb{Q}}$-rational linear combination of such functions (an argument similar to that used in the proof of Lemma 3.1 with 2 replaced by $k$ is valid). The result follows from this.

All coefficients of the individual functions on both sides of (4) are contained in a finite Galois extension $F / \mathbb{Q}$. In (4) we now take "norms" (products of Galois conjugates of $\sigma \in \operatorname{Gal}(F / \mathbb{Q}))$ to obtain an equation

$$
f^{m r}=H_{1} \cdot H_{0},
$$

where $r=[F: \mathbb{Q}], H_{1}$ and $H_{0}$ are normalized and have rational Fourier coefficients, and (thanks to Lemma 3.4) $H_{1}$ has trivial character.

Now notice that $\operatorname{div}\left(H_{0}\right)=\emptyset$. Indeed, if $\frac{2 \pi i}{N} w \in S_{2}(\Gamma)$ corresponds to the normalized PGMF $v$ upon taking logarithmic derivatives, then the cusp form $\frac{2 \pi i}{N} w^{\sigma}$ corresponds to $v^{\sigma}$ (where of course the action of Galois elements $\sigma$ is defined in the same way as above).

Since $H_{1}$ has rational coefficients and $\Gamma=\Gamma(N)$, the coefficients of $H_{1}$ must in fact be $p$-integral for almost all primes $p$ ( $\underline{\underline{S h}})$. Since $H_{1}$ is normalized, the same holds for $H_{1}^{-1}$ and therefore also for $H_{0}$ by (5). So we have arrived at the situation that $H_{0}$ is a normalized PGMF with empty divisor and rational Fourier coefficients which are $p$-integral for all but a finite number of primes $p$. By [KoM], Theorem 2, we conclude that $H_{0}=1$. Thus $f^{m r}=H_{1}$ has trivial character; i.e. the character of $f$ is of finite order. This concludes the proof of Theorem 2.1.

We turn to the proof of Corollary 2.2. Suppose first that $\chi$ is unitary. Then $f_{0}=1$, so that $f=f_{1}$ has rational Fourier coefficients by hypothesis. Therefore, $\chi$ has finite order by Theorem 2.1. This proves part (a) of the corollary. As for part (b), because the divisor $D=\operatorname{div}(f)=\operatorname{div}\left(f_{1}\right)$ is assumed to be concentrated at 
the cusps, $D$ is defined over a number field and the Manin-Drinfeld theorem tells us that $\operatorname{div}(f)$ has finite order in the divisor class group. Then $f_{1}^{m}$ has algebraic Fourier coefficients for some integer $m$; hence $f_{1}$ does too. Now Theorem 2.1]again tells us that $\chi$ has finite order. This completes the proof of part (b) of the corollary.

\section{PGMFs with ReAl Fourier COEFFicients}

In this section we briefly consider the action of complex conjugation on PGMFs. Recall Hecke's operator $K$, defined on holomorphic functions in $\mathcal{H}$ as follows ( $\mathbb{R}$, Section 8.6):

$$
f \mid K(z)=\overline{f(-\bar{z})} .
$$

If $f$ is a PGMF on $\Gamma$, then so is $f \mid K[\underline{\mathbb{R}}$, and the $q$-expansions at the infinite cusp are related as follows:

$$
f(z)=\sum a(n) q_{N}^{n}, \quad f \mid K(z)=\sum \overline{a(n)} q_{N}^{n} .
$$

Set

$$
J=\left(\begin{array}{cc}
-1 & 0 \\
0 & 1
\end{array}\right)
$$

Note that

$$
J\left(\begin{array}{ll}
a & b \\
c & d
\end{array}\right) J^{-1}=\left(\begin{array}{rr}
a & -b \\
-c & d
\end{array}\right) .
$$

In the following we will need to assume that $J$ normalizes $\Gamma$. From the last display, we see that this holds, for many congruence subgroups, e.g., $\Gamma=\Gamma(N)$, or $\Gamma_{0}(N)$. We write $\gamma^{J}=J \gamma J^{-1}$ for $\gamma \in \Gamma$. A character $\chi$ of $\Gamma$ may be 'twisted' by $J$ to yield a second character $\chi^{J}$ defined by

$$
\chi^{J}(\gamma)=\chi\left(\gamma^{J}\right) .
$$

Lemma 4.1. Assume that $J$ normalizes $\Gamma$, and suppose that the PGMF $f$ is associated with the character $\chi$. Then $f \mid K$ is associated with the character $\bar{\chi}^{J}$. In particular, $f$ has real Fourier coefficients if, and only if, $\chi=\bar{\chi}^{J}$.

Proof. For $\gamma \in \Gamma$ we have

$$
f\left|K(\gamma z)=\overline{f(-\overline{\gamma z})}=\overline{f\left(\gamma^{J}(-\bar{z})\right)}=\bar{\chi}^{J}(\gamma) f\right| K(z) .
$$

This proves the first assertion. The second follows from (6).

Suppose that $f$ is a PGMF with canonical decomposition (1) and that $\chi_{j}$ is the character associated to $f_{j}, j=0,1$. We have

$$
f \mid K=\left(f_{1} \mid K\right)\left(f_{0} \mid K\right) \text {. }
$$

By Lemma $4.1 f_{j} \mid K$ has associated character ${\overline{\chi_{j}}}^{J}$. Because $\chi_{1}$ is unitary, then so too is $\bar{\chi}_{1}^{J}$. Moreover, it is easy to see that $f_{0} \mid K$ has empty divisor. It follows from these comments that (7) is the canonical decomposition of $f \mid K$.

If now we assume that $f$ has real Fourier coefficients, then $\chi=\bar{\chi}^{J}$ by Lemma 4.1 . By the uniqueness of the canonical decomposition, we can conclude that $\bar{\chi}_{j}^{J}=\chi_{j}$ for $j=0,1$. Applying Lemma 4.1 once more, we arrive at

Lemma 4.2. Suppose that $f$ has real Fourier coefficients. Then $f_{1}$ and $f_{0}$ have real Fourier coefficients. 
The condition $\bar{\chi}_{j}^{J}=\chi_{j}$ places strong restraints on the characters $\chi_{j}$. For example, in the case of the unitary character $\chi_{1}$ we have $\chi_{1}\left(\gamma^{J} \gamma\right)=1$ for $\gamma \in \Gamma$.

Finally, notice that Lemma 4.2 applies in the context of Theorem [2.1] If we could replace the real field by a number field in the statement of the lemma, the main conjecture would follow.

\section{ACKNOWLEDGEMENTS}

The authors thank Henri Darmon and Jan H. Bruinier for useful conversations. The results of the present paper emerged from discussions during the stimulating workshop on noncongruence modular forms, organized by Ling Long and Winnie Li in August 2009, at the American Institute of Mathematics in Palo Alto.

\section{REFERENCES}

[DR] P. Deligne and M. Rapoport, Les Schémas de modules de courbes elliptiques. In: Modular Functions of One Variable. II (eds.: P. Deligne and W. Kuyk), 144-316, Lect. Not. Math., Springer, New York, 1973. MR0337993 (49:2762)

[KL] C. Kurth and L. Long, On modular forms for some noncongruence subgroups of $S L_{2}(\mathbb{Z})$. II, Bull. Lond. Math. Soc. 41, No. 4 (2009), 589-598. MR2521354 (2010i:11053)

[KM1] M. Knopp and G. Mason, Generalized modular forms, J. Number Theory 99 (2003), 1-28. MR.1957241(2003k:11061)

[KM2] M. Knopp and G. Mason, Parabolic generalized modular forms and their characters, Int. J. Number Theory 5, No. 5 (2009), 845-857. MR2553511 (2010j:11067)

[KoM] W. Kohnen and G. Mason, On generalized modular forms and their applications, Nagoya Math. J. 192 (2008), 119-136. MR2477614(2009m:11060)

[Mi] J. Milne, Algebraic geometry, Course Notes, 2009.

[R] R. Rankin, Modular Forms, Cambridge University Press, Cambridge, 1977. MR 0498390 (58:16518)

[Sc] A. J. Scholl, Fourier coefficients of Eisenstein series on non-congruence subgroups, Math. Proc. Camb. Phil. Soc. 99 (1986), 11-17. MR809492 (86m:11028)

[Sh] G. Shimura, Introduction to the arithmetic theory of automorphic forms, Iwanami Shoten and Princeton University Press, 1971. MR0314766 (47:3318)

[W] M. Waldschmidt, Nombres transcendents et groupes algébraiques, Astérisque 69-70 (1979). MR570648 (82k:10041)

Mathematisches Institut der Universität Heidelberg, INF 288, D-69120 Heidelberg, GERMAnY

E-mail address: winfried@mathi.uni-heidelberg.de

Department of Mathematics, University of California at Santa Cruz, Santa Cruz, CALIFORNIA 95064

E-mail address: gem@cats.ucsc.edu 